$7 \quad{ }^{1}$ Neuroscience Graduate Program, University of Southern California, Los Angeles, CA 90089-

8 2520, USA

$9 \quad{ }^{2}$ Biokinesiology and Physical Therapy, University of Southern California, Los Angeles, CA

10 90089-9006, USA

12 Correspondence: Nicolas Schweighofer: schweigh@usc.edu

138 Figures. Abstract: 248 words. Introduction 536 words. Discussion 1488 words.

\title{
16 Acknowledgements
}

17 We thank Jun Izawa for helpful discussions on models of motor adaptation and Raphael

18 Schween for valuable comments on a previous draft. This work was funded by grants NSF BCS

19 1031899, NIH R01 HD065438 and R56 NS100528 to NS. 


\section{Abstract}

21 The central nervous system predicts the consequences of motor commands by leaning multiple

22 internal models of external perturbations and of the body. It is not well understood, however,

23 how new internal models are created. Here, we propose a novel computational model of motor

24 adaptation in which a stochastic Bayesian decision-making process determines whether i) a

25 previously updated expert perturbation model is recalled and updated, ii) a novice model is

26 selected and is updated into a new expert, or iii) the "body" model is updated. Results from

27 computer simulations provide insights into various and contradictory experimental data on

28 savings and error-clamp, and predicts qualitative individual differences in adaptation. We

29 verified these predictions in a visuomotor adaptation experiment in which we varied the

30 perturbation amplitudes as well as the amount of noise added to perturbation, and added

31 "trigger" trials in the error-clamp condition. Single trigger trials led to largely qualitatively

32 different behavior and can therefore be used to probe individual differences in memory updates

33 between "one-model" and "two-model” learners. "One-model” learners continuously update the

34 body model, showing no savings during re-adaptation to the perturbation, and gradual decay

35 during error clamp. In contrast, "two-model” learners switch between an updated expert model

36 and the body model, showing large savings during re-adaptation and stochastic lags during error

37 clamp. Our results thus support the view that motor adaptation belongs to the general class of

38 human learning according to which new memories are created when no existing memories can

39 account for discontinuities in sensory data. 


\section{Short summary/significance}

42 When movements are followed by unexpected outcomes, such as following the introduction of a

43 visuomotor or force field perturbation, or the sudden removal of such perturbations, it is unclear

44 whether the central nervous system updates existing memories or creates new memories. Here,

45 we propose a novel model of adaptation, and investigate, via simulation and behavioral

46 experiments, how the amplitude and schedule of the perturbation, as well as the characteristics of

47 the learner, lead to updates of existing memories or creation of new memories. Our results

48 provide insights into a number of puzzling and contradictory experimental data on savings and

49 error-clamp, as well as large qualitative individual differences in adaptation. 


\section{Introduction}

53 It is now well accepted that the central nervous system (CNS) predicts the consequences of

54 motor commands by leaning multiple models of tools or external perturbations (Wolpert and

55 Kawato, 1998; Krakauer et al., 1999; Lee and Schweighofer, 2009), and by learning internal

56 models of the body (Cothros et al., 2006; Kording et al., 2007; Kluzik et al., 2008). Forward

57 models are updated to minimize sensory prediction errors, i.e., the errors between sensory

58 outcomes and predictions (Mazzoni and Krakauer, 2006; Taylor and Ivry, 2011; Lee et al.,

59 2018). It is thought that if the prediction error is small for one model, then this model will be

60 selected to determine subsequent motor commands and will be further updated if needed

61 (Wolpert and Flanagan, 2001).

62 It is not well understood, however, how the CNS creates new internal models (Shadmehr and

63 Mussa-Ivaldi, 2012). When movements are followed by unexpected outcomes, such as due to a

64 visuomotor or a force field perturbation, should the CNS update an internal model of the body,

65 should it update one or more perturbation models, or should an entirely new internal model be

66 created?

67 Here, to address this question, we propose a new model of motor adaptation based on a bank of

68 Kalman filters, along the lines of a recent model of visual memory (Gershman et al., 2014). The

69 model contains three types of internal models involved in motor adaptation: 1) a dedicated body

70 (or "baseline") model (Berniker and Kording, 2011); 2) "expert" perturbation models that have

71 been previously adapted (Kawato and Wolpert, 1998; Lee and Schweighofer, 2009; Berniker and

72 Kording, 2011); and 3) "novice" unspecific models. As in previous Bayesian models, the

73 decision to select and update a particular internal model when facing a perturbation is based on 
74 the models' prediction errors. If a perturbation is small relative to uncertainty of the baseline

75 model, or if the perturbation is gradually increased, the baseline model is selected and its

76 memory is overwritten. If a relatively large perturbation is re-introduced, or if it is similar to a

77 perturbation encountered in the past, one of the existing expert perturbation models will be

78 selected, leading to the recall of its protected memory. If a new large perturbation is introduced

79 and if it does not match prediction of any existing internal models, then a new novice model is

80 selected and updated. After sufficient training, this novice model becomes a new expert. Besides

81 perturbation amplitude, perturbation noise, motor noise, or individual differences in baseline

82 model uncertainty will influence the effect of the prediction error on model selection and update.

83 We tested these predictions in a visuomotor adaptation experiment in which we varied the

84 perturbation amplitudes as well as the amount of noise added to perturbation across different

85 experiment groups. At the end of repeating perturbation and washout blocks, a visual error-

86 clamp block was introduced to observe spontaneous and stochastic changes in adaptation. In

87 addition, we inserted two trigger trials (each is a single perturbation trial) in the error-clamp

88 block to test whether a learner has truly developed a new expert model or simply updated the

89 baseline model. The large individual differences in after-effects, savings, and error-clamp can all

90 be accounted for by the uncertainty of the learner in the baseline model.

92 Materials and Methods

\section{Computational model}

94 Our model is based on previous Bayesian models of motor learning known as a mixture of

95 experts (Jordan and Jacobs, 1991; Ghahramani and Wolpert, 1997; Wolpert and Kawato, 1998; 
96 Haruno et al., 2001). Specifically, we extend previous models of adaptation based on the Kalman

97 filter (Korenberg and Ghahramani, 2002; Berniker and Kording, 2011) to a model that contains a

98 bank of Kalman filters, along the lines of the model of Gershman et al. (2014) for visual

99 memory. We thus assume that the experts maintain, and udpate, both a mean perturbation

100 estimate and an uncertainty around this estimate. Before being selected for training, these expert

101 models are "novice" with high uncertainty, indicating their non-specificity. In addition, we

102 included a special baseline model (similar to the "body model" in Berniker and Kording (2011))

103 that estimates the mean perturbation to be zero with low uncertainty and that has a high prior of

104 being selected (Figure 1A).

105 As in previous models of visuomotor adaptation, e.g., (Izawa and Shadmehr, 2011), the motor

106 command represents the hand movement direction. On trial $t$, the learner generates a motor

107 command $u_{t}$ to reach a target $t_{t}$. Here, we assume that the target is located at the angle 0 , that is,

108 the forward direction, without loss of generality. Visual feedback of the hand $h_{t}$ is determined

109 differently in non error-clamp trials (i.e., baseline, perturbation, and washout) and error-clamp

110 trials, in which feedback is independent of actual performance:

$111 h_{t}=\left\{\begin{array}{cc}u_{t}+p_{t}+n_{t}^{p}, & \text { non error clamp trials } \\ n_{t}^{e c}, & \text { error clamp trials }\end{array}\right\}$,

112 where $p_{t}$ is the perturbation at time $t$, and $n_{t}^{p} \sim N\left(0, \sigma_{p}^{2}\right)$ and $n_{t}^{e c} \sim N\left(0, \sigma_{e c}^{2}\right)$ are noise sources

113 added to the perturbation or to the error clamp, respectively.

114 The estimate of the perturbation $\hat{p}_{t}$ can be given by the weighted predictions from the $\mathrm{N}$ models

115 or by the model with the largest weight, in a form of winner-take-all. Although both methods can

116 produce similar results, we chose the winner-take-all approach because it is more robust to

117 changes in parameters. Thus, the overall prediction at time $\mathrm{t}$ is given by: 
$118 \hat{p}_{t}=\hat{p}_{t}^{i}, \quad$ with $i=\operatorname{argmax}_{j}\left(w_{t}^{j}\right) \quad$,

119 where $w_{t}^{i}$ is weight of model $i$, with $\sum_{i}^{N} w_{t}^{i}=1$

120 In order to reach the target at location 0 , we assume that subjects generate motor command $u_{t}$

121 that compensates the estimated perturbation $\hat{p}_{t}$ :

$122 u_{t}=-\hat{p}_{t}+n_{t}^{u}$

123 where $n_{t}^{u} \sim N\left(0, \sigma_{u}^{2}\right)$ is a motor noise term. Receiving the efferent copy of the motor command,

124 each internal forward model independently predicts the sensory feedback from its own

125 perturbation estimate:

$126 \hat{h}_{t}^{i}=u_{t}+\hat{p}_{t}^{i}$

127 The sensory prediction error for each model is given by:

$128 \varepsilon_{t}^{i}=h_{t}-\hat{h}_{t}^{i}$

129 The weights (the "responsibility signal" in previous models such as the MOSAIC models

130 (Wolpert and Kawato, 1998; Haruno et al., 2001; Doya et al., 2002; Bertin et al., 2007)), are

131 given by the posterior probability of the models, given the visual feedback:

$w_{t}^{i}=\rho^{i} e^{-\frac{\varepsilon_{t}^{i^{2}}}{2 \widehat{S}_{t}^{i}}} / \sum_{j=1}^{N} \rho^{j} e^{-\frac{\varepsilon_{t}^{j^{2}}}{2 \widehat{S}_{t}^{j}}}$

133 where $\rho^{i}$ is a constant prior weight representing prior belief that each model being true in the

134 absence of feedback, and $\hat{S}_{t}^{i}$ is the uncertainty of a model $i$ around its mean estimate $\hat{p}_{t}^{i}$. Note

135 that the term in the exponents assumes a Gaussian likelihood function for observations based on 136 predictions. 
137 The predictions and uncertainties are updated accorded to standard Kalman filter equations, e.g.,

138 (Bishop and Welch, 2001). However, the type of update for each model in the bank of Kalman

139 filters depends on the weights. When a model is selected, it is updated according to the

140 measurement update equations of the Kalman filter:

141 for $i=\operatorname{argmax}_{j}\left(w_{t}^{j}\right)$ :

$142\left\{\begin{array}{c}K_{t}^{i}=\hat{S}_{t-1}^{i} /\left(\hat{S}_{t-1}^{i}+\sigma_{m}^{2}\right) \\ \hat{p}_{t}^{i}=a^{i} \hat{p}_{t-1}^{i}+K_{t}^{i} \varepsilon_{t}^{i} \\ \hat{S}_{t}^{i}=\left(1-K_{t}^{i}\right) \hat{S}_{t-1}^{i}\end{array}\right.$,

143 where, $0<a_{i} \leq 1$ are decay rate parameters and $\sigma_{m}$ a measurement noise parameter. When a

144 model is not selected, it is updated according to the time update equations of the Kalman filter:

145 for $j \neq i$ :

$146\left\{\begin{array}{c}\hat{p}_{t}^{i}=a_{i} \hat{p}_{t-1}^{i} \\ \hat{S}_{t}^{i}=a^{i 2} \hat{S}_{t-1}^{i}+\sigma_{s}^{2}\end{array}\right.$,

147 where $\sigma_{s}$ a state noise parameter.

\section{Theoretical predictions}

150 For simplicity, we only consider here a computational model that accounts for adaptation to a

151 single perturbation, with two internal models (Figure 1B): Initially, the model contains a baseline

152 model that estimates the mean perturbation to be zero with low uncertainty, indicating its

153 specificity, and a "novice" model also estimates the mean perturbation to be zero initially but

154 with larger uncertainty, indicating its non-specificity. 
155 The model makes clear predictions that can be tested in simulations and behaviorally. First, it

156 predicts that a large initial perturbation leads to rapid subsequent changes in performance, such

157 as savings and short aftereffects following environmental changes, whereas a small or gradual

158 initial perturbation creates no such rapid changes, but smooth and gradual changes. The former is

159 explained by switching between two expert models while the latter is the result of overwriting

160 the baseline model. More specifically, if an initial perturbation is sufficiently large, i.e., away

161 from the decision boundary defined by the equal weights of the baseline and the novice model,

$162 w_{1}=w_{2}=0.5$ (vertical dashed lines in Figure 1B), the novice model is selected and updated,

163 while the baseline model is protected. After a number of trials, the novice model becomes a new

164 expert: the mean estimate of the perturbation is accurate and the uncertainty becomes small. As a

165 result, the learner can now switch rapidly between these two expert models in subsequent

166 unlearning and relearning blocks, allowing rapid changes of behavior upon environmental

167 changes, expressed as savings in relearning and short or no aftereffects in washout. We call such

168 a learner a "two-model learner". On the contrary, following a perturbation within the decision

169 boundaries of the baseline model, as would be encountered with a small or gradual perturbation,

170 the baseline model is selected and updated with no update of the novice model. This "one-model

171 learner" will be updated in subsequent washout and relearning blocks of the same amplitude,

172 making transitions slow and gradual, with no savings. In washout, this model will decay

173 gradually back to baseline via trial-by-trial decay.

174 Second, the model predicts that once a learner has formed two models, switching can occur

175 between the two models in error-clamp. This is because what triggers model switching is the

176 sensory prediction error (equation 6, right panel), but not the performance error, which is

177 clamped to around zero in error-clamp. Thus, assuming that the hand direction is near the 
178 adapted direction after a training block, it can show nor or little decay with a lag: the hand

179 direction hovers near the adapted directions until a sudden drop towards baseline. However, high

180 levels of motor noise or experimentally induced perturbation noise increases the probability of

181 yielding a small sensory prediction error for the baseline model, leading to a greater weight for

182 this model, and therefore model switching. Once this happens, performance drops quickly

183 towards baseline. Thus, the model predicts that a sudden drop or rise in performance can occur

184 stochastically in error-clamp, with higher chance with a high level of either perturbation or motor

185 noise. A "rise" can also occur when switching from the baseline to the perturbation model,

186 although this should be a less frequent event than the drop because of the passive memory decay

187 and because of the greater prior assigned to the baseline model.

188 Next, because the model has qualitatively different modes of operation, single "trigger" trials in

189 the clamp condition can lead to largely qualitatively different behavior when the performance

190 has returned to zero: a trigger trial will cause a sudden rise to the learned level for two-model

191 learners, whereas it will cause only a transient change that decays away quickly for one-model

192 learners.

193

194 Simulations and experimental design

195 We designed five different sets of simulations to test the above predictions quantitatively. We

196 then performed experiments and data analyses that closely matched the simulation protocols for

197 the first four sets. The simulations and experiments comprised a baseline block, adaptation

198 blocks, washout blocks, and an error-clamp block (Figure 2A). 
199 First, we simulated the effects of large versus small perturbations on subsequent behaviors in

200 unlearning and relearning blocks with a large perturbation (20 degrees) and with a small

201 perturbation (10 degrees). To test for possible time-effect of washout and savings, conditions

202 were simulated with repeated learning and unlearning blocks: baseline (20 trials), learning and

203 unlearning block 1 (60 perturbation trials followed by 40 washout trials), learning and unlearning

204 block 2 (50 perturbation trials followed by 20 washout trials), learning and unlearning block 3

205 (30 perturbation trials followed by 40 washout trials), and learning block 4 (50 perturbation

206 trials, followed by 120 error clamp trials) (see Figure 2B).

207 Second, we simulated the effects of large versus small noise levels in error-clamp with large

208 perturbations (20 degrees). The schedule for these simulations was the same as above, but two

209 different noise levels were added to the perturbation. We predicted that the large-noise condition

210 would exhibit smaller lags until decay (i.e., earlier drops from the adapted state to baseline), and

211 the small-noise condition would exhibit longer lags. A density plot of hand directions in the

212 error-clamp block would show distinct distributions for the two conditions: the small-noise

213 condition would have two dominant peaks the distribution of hand directions, one around the

214 learned direction and the other around zero. In contrast, the large-noise condition would have a

215 single dominant peak near zero. The model also predicts that one-model learners, such as found

216 in the small-perturbation condition, would exhibit smooth and intermediate hand distributions

217 between the adapted level and the baseline level in error-clamp, because gradual passive decay

218 occurs in this case, and not model switching.

219 Third, in addition to the error-clamp simulation described above, we also simulated the effect of

220 two separate trigger trials during error-clamp. The prediction is that if a learner has formed an

221 expert model, then a trigger trial would cause a sudden jump to the learned level if the hand 
222 direction were already close to zero before the trigger, and would stay in this up state for several

223 trials following this jump. Otherwise, if the hand were still around the learned level, then a

224 trigger would make no change because it is indistinguishable from the error-clamp trials.

225 Fourth, to simulate individual differences in adaptation, washout, savings, and error-clamp, we

226 made a change to a single parameter, the baseline model uncertainty (and associated minimum

227 uncertainty $\hat{S}_{\text {min }}$ ), with a first group having a larger uncertainty than the second group.

228 Finally, we simulated the effects of gradual versus abrupt perturbation, using paradigms akin to

229 those used in Roemmich and Bastian (2015). We tested for savings in a second abrupt adaptation

230 block of 100 trials that is preceded by either a gradual (100 trials), abrupt (100 trials), or short

231 adaptation (20 trials) block.

\section{Experimental Methods}

234 Fifty-four subjects (22.5 \pm 3.8 years old, 20 males and 34 females) participated in the study, which

235 was approved by the Institutional Review Board at the University of Southern California, after

236 signing an informed consent. Subjects sat in front of a device that matched hand space with

237 visual space via a mirror, and were instructed to hold a stylus pen moving on a digitizer tablet

238 (Wacom Intuos 7). Head and trunk movements were limited via a chin-rest. The experiment took

239 place in a dark room, and the mirror-obscured view of the forearm and hand. A cursor (red dot of

$2401.2 \mathrm{~mm}$ radius) representing the tip of the pen was displayed on the mirror. Before the start of

241 each trial, subjects were instructed to position the cursor inside a home circle of a $3 \mathrm{~mm}$ radius

242 (about $36 \mathrm{~cm}$ away from the subject's torso). We used a polar coordinate system centered on the

243 home circle, with $0^{\circ}$ defined as the forward direction and positive direction as clockwise 
244 deviation. Subjects were instructed to perform an outward shooting movement toward a circular

245 target of $3^{\circ}$ radius. The target appeared at a pseudorandom location each trial, within $5^{\circ}$ around

246 the center of a $120^{\circ}$ arc that was $10 \mathrm{~cm}$ away from the starting position. Subjects were told to

247 initiate a shooting movement as soon as a target appeared and stopped after crossing the arc. A

248 red dot representing a cursor disappeared when the pen tip moved farther than $3 \mathrm{~cm}$ from the

249 starting position. When the pen tip crossed the arc, the red dot was displayed on the crossed-

250 point and remained there for $1 \mathrm{~s}$. Subjects were encouraged to keep movement duration between

$251100 \mathrm{~ms}$ and $300 \mathrm{~ms}$, where movement duration was defined as a time interval from the moment

252 when the cursor disappeared to the moment when the cursor crossed the target arc. The

253 messages "Too Slow" or "Too Fast" were displayed when movement duration was out of this

254 range. After each shooting movement, subjects then performed an inward movement to the home

255 circle, during which only the radial location of the cursor was available.

256 After a familiarization session of 80 trials with no perturbation, the main experimental schedule

257 consisted of repeated learning and washout blocks followed by an error-clamped block. The

258 visuomotor perturbation rotated the cursor position counterclockwise by a given angle with

259 respect to the starting position. The experimental design was closely aligned to the design of the

260 simulations. We randomly assigned subjects into one of five different conditions (Figure 2B): 1a,

$2612 \mathrm{a}, 3,1 \mathrm{~b}$, and $2 \mathrm{~b}-$ see below for a condition description. In all conditions, the experiment

262 schedule consisted of learning blocks was the same order and number of trials as in simulations

263 (see above and Figure 2B). The block lengths were adjusted in pilot tests to allow performance to

264 plateau in each test. The numbers of trials in the learning and unlearning blocks were purposely

265 varied to prevent predictable periodicity in the experiment. 
266 Conditions 1a, 2a, and 3 differed in either rotation angles (Condition 1 vs. Condition 3), or in

267 Gaussian noise levels added to the cursor location (Condition 1 vs. Condition 2). Condition 1a

$268(\mathrm{n}=11)$ : large perturbation $\left(20^{\circ}\right)$ and small noise level $\left(\operatorname{std}\right.$ of $\left.0.5^{\circ}\right)$. Condition $2 \mathrm{a}(\mathrm{n}=11)$ : large

269 perturbation $\left(20^{\circ}\right)$ and large noise level $\left(\operatorname{std}\right.$ of $\left.4.0^{\circ}\right)$. Condition $3(n=11)$ : small perturbation $\left(10^{\circ}\right)$

270 and small noise level ( $s t d$ of $0.5^{\circ}$ ). Conditions $1 \mathrm{~b}$ and $2 \mathrm{~b}$ were identical to conditions $1 \mathrm{a}$ and $2 \mathrm{a}$,

271 respectively, except for two "trigger trials" inserted at half and three quarters of error-clamp,

272 respectively. Trigger trials were simply rotation trials, identical to those in the learning condition

273 (i.e., $20^{\circ}$ rotation). In the error-clamp block of all conditions, cursor feedback was independent of

274 actual hand directions, and was sampled from a pre-determined Gaussian distribution of mean $0^{\circ}$

275 and standard deviation $0.5^{\circ}$ or $4.0^{\circ}$, depending on the condition noise level. All subjects in each

276 condition received exactly the same rotation sequence.

278 Data Analysis

279 We predicted that the large-perturbation condition exhibits quick changes after the initial

280 learning block whereas the small-perturbation condition is accompanied by slow and gradual

281 changes in all blocks, resulting in large time constants. To test these predictions, we fit

282 performance in each block with an exponential function,

$$
f\left(t_{B}\right)=A+B\left(1 \quad e^{t_{B} /}\right)
$$

284 where $t_{B}$ is a trial number counted from the start of each block, A an amplitude parameter, and $\tau$

285 the time constant. Because the distributions of time constants between groups were not normally

286 distributed, we used Friedman test and Wilcoxon-Mann-Whitney Test to test for differences. 


\section{Simulation parameters}

289 In all simulations, hand direction was modeled between 0 and 1, and then scaled by 20 degrees to

290 match experimental data. We determined a single set of parameters that could replicate all

291 experimental results qualitatively: standard deviations of both sensory noise $\sigma_{p}$ and error-clamp

292 noise $\sigma_{e c}$ (equation 1 ) were 0.2 for the large-noise condition and 0.025 for the small-noise

293 condition. The motor noise standard deviation $\sigma_{u}$ was 0.15 , the measurement noise $\sigma_{m}$ was 1 and

294 the state noise $\sigma_{s}$ was 0.01 . The prior constant of the baseline model $\rho^{1}$ was 0.95 and that of the

295 novice model $\rho^{2}$ was 0.05 . Initial mean perturbation values were 0 . The initial uncertainties for

296 the baseline and novice (perturbation) model were set to 0.2 and 0.6 . In simulations aimed at

297 studying between-subject variability, we increased the initial uncertainty for the perturbation

298 model (and corresponding minimum uncertainty for this model) to 0.35 .

299 Note that to be able to qualitatively reproduce the data, we needed to make two assumptions.

300 First, retention parameters $a$ were given different values for the perturbation model and for the

301 baseline and: 0.999 for the perturbation model and 0.98 for the baseline model (See Discussion

302 for justification of this choice). Second, we needed to introduce a minimum value $\hat{S}_{\min }$ to

303 maintain numeric stability, and took the baseline model uncertainty equal to this minimum value.

304 The minimum uncertainty $S_{\min }$ was 0.2 . 


\section{Results}

\section{Example of simulations illustrating effects of perturbation sizes and noise levels}

310 We first show some examples of simulations for illustrations. In later sections, we present

311 average results over multiple runs. The first column of Figure 3 shows examples of hand

312 directions in the five experimental conditions. The effect of perturbation sizes can be seen by

313 comparing hand direction in the large perturbation conditions $\left(20^{\circ}\right.$; conditions $\left.1 \mathrm{a}, 2 \mathrm{a}\right)$ to that in

314 the small condition $\left(10^{\circ}\right.$; condition 3 ; last row $)$. In the large perturbation conditions, hand

315 direction exhibited little aftereffect in washout, large savings in relearning (i.e., one-trial rise),

316 and abrupt switches in error-clamp after varying decay lags or after trigger trials. On the other

317 hand, the small perturbation condition was accompanied by gradual and smooth changes in

318 washout and in relearning blocks, indicating strong aftereffect and no or little savings. Similarly,

319 decay in error-clamp was gradual.

320 The effect of noise levels can be illustrated by comparing examples of simulations with low

321 levels of noise (condition 1a and $1 \mathrm{~b}$ ) to simulations with high levels of noise (conditions $2 \mathrm{a}$ and

322 2b), in which each pair has identical parameters except for the noise level. Simulations with high

323 levels of noise show earlier switching to baseline in error-clamp than with low levels.

324 The second column of Figure 3 displays the estimated perturbation mean $\hat{p}_{i}$, and uncertainty $\hat{S}^{i}$

325 for the baseline and perturbation models. In the large perturbation and small noise conditions

326 (conditions 1a and 1b), the novice model was selected in the initial learning block and its mean

327 estimate increased to approximate the true perturbation level, while its uncertainty

328 simultaneously decreased. The baseline model was practically unchanged. In contrast, in the

329 small perturbation condition (conditions 3), the baseline model updated its estimate of the 16 
330 perturbation up and down each time the environment changed. In contrast, the novice model

331 remained unchanged in this condition, making this learner a one-model learner.

332 The third column of Figure 3 shows the sum of the likelihoods for both models. As can be seen

333 in the first four conditions, the likelihood of the perturbation model increased as this model better

334 predicted the perturbation. This increase was due to both the mean prediction converging to the

335 perturbation level and the decrease in model uncertainty, as the model was selected (see equation

336 7).

337 The fourth column of Figure 3 visualizes the weight map of the perturbation model,

338 superimposed with the weight for this model. As a reminder, because only two models were

339 simulated, the decision boundary is given by $w_{1}=w_{2}=0.5$. In error-clamp, when the

340 perturbation model is near the adapted state, the prediction error is small, and the perturbation

341 model is continuously updated. However, memory decay, perturbation noise, or motor noise, can

342 lead a large prediction error for the perturbation model, yielding a rapid switch to the baseline

343 model. Thus, because the probability of transition was higher for the high perturbation noise

344 level, the lags in error clamps were shorter on average than in the low noise conditions (compare

345 lags in conditions $2 \mathrm{a}$ and $2 \mathrm{~b}$ to those in conditions $1 \mathrm{a}$ and $1 \mathrm{~b})$. Note that, because of the time-

346 dependent decay of the perturbation estimate, and the higher prior given to the baseline model,

347 the direction of transition is asymmetric, with a higher probability of switching from the

348 perturbation model to the baseline model than in the other direction.

349 The triggers (vertical red lines in the first columns of conditions $1 \mathrm{~b}$ and $2 \mathrm{~b}$ ) can act as a cue to

350 signal switching back to the perturbation model when the learner has already returned to the

351 baseline level before a trigger trial. This is shown following the first trigger trials in conditions

$3521 \mathrm{~b}$ and $2 \mathrm{~b}$. Such switching happens when a single trigger is sufficient to reduce the sensory 
353 prediction error for the perturbation model. This transition, however, is probabilistic due to the

354 motor and perturbation noises. Note that if trial-by-trial decay has sufficiently decreased the

355 memory of the perturbation at the time of trigger, there is large prediction error for this model

356 after the trigger, which will therefore be ineffective (results not shown)

\section{and simulation results}

Figure 4A shows subject-averaged adaptation data for experimental conditions $1 \mathrm{a}$ and $1 \mathrm{~b}\left(20^{\circ} /\right.$ $0.5^{\circ}$; top row) combined and for condition $3\left(10^{\circ} / 0.5^{\circ}\right.$; bottom row $)$. Figure $4 \mathrm{~B}$ shows the mean time constants fitted to the exponential curve (equation 9) within each block across subjects for both learning and washout blocks. Subjects in the large perturbation conditions $\left(20^{\circ}\right)$ showed savings, i.e., significant decrease of time constants in the re-learning blocks (LB2, LB3, and LB4), compared to the initial learning block (LB1; Friedman test; $\quad{ }^{2}=34.6, p=1.5 \quad 10^{7}$ for conditions $1 \mathrm{a} / \mathrm{b} ; \quad{ }^{2}=13.1, p=4.510^{3}$ for conditions 2 ). Post-hoc analysis revealed that savings occurred in all re-learning blocks of conditions 1, with smaller time constants compared pair; $Z=3.92, p=1.1 \quad 10^{5}$ for LB1-LB3 pair; and $Z=3.92, p=1.1 \quad 10{ }^{5}$ for LB1-LB4 pair).

371 perturbation condition $\left(10^{\circ}\right.$; condition 3; Friedman test; $\left.{ }^{2}=2.3, p=0.51\right)$, indicating a lack of

372 savings. In addition, the overall time constants in washout blocks of conditions 1 were 
373 significantly smaller than those in condition 3 (Wilcoxon-Mann-Whitney Test;

$374 Z=2.6, p=0.029)$.

375 In simulations (Figure 4C), the large-perturbation condition produced a median time constant of

37616 trials for the first learning block (LB1), followed by small time constants less than 1 trial for

377 the subsequent learning blocks (LB2, LB3, and LB4). The washout blocks produced an averaged

378 median time constants of less than 1 trial across the three washout blocks (WB1, WB2, and

379 WB3). These short time constants were a direct consequence of model switches upon the

380 perturbation change. On the other hand, the small-perturbation condition produced median time

381 constants between 10 and 12 trials for all four learning blocks and median time constants

382 between 11 and 15 for all three washout blocks. These long time constants were a consequence

383 of the baseline model being continuously updated each time the environment changed.

385 Decay in error-clamp: Experimental and simulation results

386 In Figure 5, we show both condition-averaged and individual hand direction in error clamps.

387 Although averaged data suggest a continuous and gradual decay, between-subject variability was

388 large. For instance, for subjects in condition 1a (large perturbation and small noise; Figure 5A),

389 the average between-subject standard deviation of hand direction in learning and unlearning

390 blocks was $3.9^{\circ}$, whereas that in the error-clamp block it was $8.9^{\circ}$. Larger inter-subject variability

391 in error-clamp indicates that dynamics of unlearning in error-clamp may not follow a simple

392 decay. Instead, examination of individual data shows various patterns in error-clamp, with most

393 subjects showing a lag, as predicted by our simulations (see Figure 3). For example, subjects 1,

3945 , and 10 in condition 1a showed little or no decay, with hand direction above $10^{\circ}$ for the whole 
395 duration of the clamp. In contrast, subjects 2,7 , and 8 showed a sudden drop after varying lags

396 following the onset of error-clamp. Finally, subjects 4, 6, 9, and 11 showed rather gradual decay.

397 A density plot of hand distribution in error-clamp for all these subjects (Figure 5D, red curve)

398 shows two peaks centered near 0 and 20 degrees. Thus, overall, the hand direction of subjects in

399 condition 1a remained near the perturbation angle of $20^{\circ}$ for a relatively large number of trials,

400 and then switched abruptly to near $0^{\circ}$, with few trials between these two angles.

401 Average hand direction in condition 2a appears to show faster return to baseline in error-clamp

402 trials compared to condition 1a (Figure 5B), as predicted in simulations. Here again not all

403 subjects followed a simple gradual decay. Subjects 12, 16, 17, 19, and 20 in particular exhibited

404 sudden drops, with subjects 12,16 , and 20 switching back to near $20^{\circ}$ spontaneously, resulting in

405 an oscillatory pattern. Note that among those who showed a gradual decay, subjects 13,15 , and

40621 started near $10^{\circ}$ at the onset of error-clamp; we will discuss a possible cause for this behavior

407 below. A density plot of hand distribution for subjects of condition 2a (Figure 5D, blue curve)

408 shows a single peak centered near 0 degree with a fat right tail. Thus, overall, the hand direction

409 of subjects in this condition also showed sudden switches between perturbation angle and

410 baseline, but such switches occurred earlier than condition 1a with low noise, with occasional

411 spontaneous return back to near $20^{\circ}$.

412 Condition 3 (small perturbation and small noise; Figure 5C) shows an overall trend of gradual

413 decay and occasional oscillations. The distribution of hand directions suggests that decay was

414 gradual and slow: whereas the density plot in the large perturbation conditions 1a and 2a shows

415 at least one peak near $0^{\circ}$, the distribution of hand direction in condition 3 has a single peak

416 around $8^{\circ}$ (Figure 5D, green curve). This suggests that there was no abrupt change, and most

417 subjects did not decay completely to $0^{\circ}$ (note however that in this condition, the starting angle in 
418 error-clamp was around $10^{\circ}$, and thus trial-by-trial noise made it difficult to distinguish switches,

419 if any, from noise).

420 For comparison, the right panel of Figure 5D shows the corresponding distributions of hand

421 directions from multiple independent simulation runs of each condition. Compared to data,

422 simulation results show relatively narrower distributions, but they overall replicate the general

423 patterns of density of hand directions in the experimental data, including shapes of distributions

424 and location of peaks in the three conditions.

426 Trigger trials and individual differences in adaptation: Experimental and

\section{7 simulation and results}

428 In order to investigate whether a learner had formed two models, i.e., a baseline and a

429 perturbation model, we introduced two trigger trials during error-clamp both in experiments and

430 in simulations of conditions $1 b$ and $2 b$ (Figure 6).

431 Condition-averaged hand direction in error-clamp showed instantaneous responses to the triggers

432 (i.e., sudden jumps in hand direction), both in conditions $1 \mathrm{~b}$ and $2 \mathrm{~b}$. The response appears

433 sustained for a number of error-clamp trials thereafter (Figure 6A and 6B, left panels). However,

434 the averaged hand direction indicates that the responses to triggers were, on average incomplete

435 in magnitude, i.e., smaller than $20^{\circ}$. This is because not all individuals responded to the trigger;

436 indeed, three patterns can be observed. First, when the hand direction was near $0^{\circ}$ when a trigger

437 was presented, a majority of subjects (subjects $34,38,39,41,42,43$, and 44 of condition 1b; 45,

$43846,49,51,53$ and 54 of condition 2 b) showed immediate jumps in response to the trigger to

439 angle values near the adapted state. In contrast, and as expected when the hand direction was 
near $20^{\circ}$ at the time of trigger, there was no effect (see subject 37,38 of condition $1 \mathrm{~b}$ and subject

44145,49 , and 54 of condition $2 b$ ). These first two patterns were predicted by our model - compare

442 results for these subjects with the model's response to trigger in Figure 3A.

443 However, a third pattern is also apparent: subjects with hand direction near $0^{\circ}$ at the time of

444 trigger who did not respond to the trigger (subjects 35 and 36 of condition $1 b$; subjects 47, 48,

44550 , and 52 of condition $2 \mathrm{~b}$ ). We note that those non-responding subjects appeared to have started

446 the error-clamp block with a lower level of adaptation than those who responded. We therefore

447 hypothesized that subjects who fully adapted to the perturbation formed a new perturbation

448 model, that is, were two-model learners. In contrast, subjects who did not fully adapted to the

449 perturbation only updated the baseline model, that is, were one-model learners.

450 To test this hypothesis, we divided subjects in conditions $1 \mathrm{~b}$ and $2 \mathrm{~b}$ between "full-learners", as

451 defined by subjects whose asymptotic adaptation angle in the last learning block (LB4) was

452 greater than or equal to $80 \%$ of the full angle (so more or equal to $16^{\circ}$ ), and "partial-learners" if

453 below $16^{\circ}$. Figure 6C (left panel) shows a strong negative correlation between the hand direction

454 before the trigger trials and the amount of hand change after the trigger trials for the full-learners.

455 The slope of -0.86 , which is close to -1 , indicates that the hand direction switched to around $20^{\circ}$

456 after the trigger, independently of hand direction before the trigger. In contrast, the partial-

457 learners failed to show such a relationship. As can be seen by the cluster of blue circles on the

458 bottom left of the figure, most subjects who had a lower adaptation level at the end of adaptation

459 failed to respond to the trigger trials.

460 Figure 6C (right panel) shows simulation results that account for these patterns of response to

461 triggers for 20 simulated subjects. Two sub-groups of subjects were simulated: a sub-group with

462 default baseline uncertainty (and with the associated minimum uncertainty) of 0.2 , the two- 
model learners; and a sub-group with a broader baseline uncertainty of 0.35 , the one-model

464 learners (Note that because the model parameters are scaled to a maximum perturbation of 1 , the

465 two-model and one-model learners baseline model uncertainty correspond to $4^{\circ}$ and $7^{\circ}$,

466 respectively). The sub-group with the default, more narrow, baseline uncertainty developed two

467 models during adaptation, and responded to triggers in clamp, as shown in Figure 3. In contrast,

468 the sub-group with the broader baseline model uncertainty did not develop a new perturbation

469 model, and therefore behaved as the one model learner in the last row of Figure 3 (but of course,

470 trying to adapt to a $20^{\circ}$ instead of a $10^{\circ}$ perturbation). Thus, in our paradigm, $20^{\circ}$ appears to be a

471 large perturbation for most subjects, leading to the development of a new model. For several

472 other subjects however, the same $20^{\circ}$ perturbation appears to only warrant the update of the

473 baseline model.

474 Based on the savings results of large and small perturbation shown in Figure 4, we then

475 conjectured that the subjects who showed low levels of adaptation at the end of the last

476 adaptation block, and therefore who, for the most part, failed to respond to triggers, would show

477 little or no savings in relearning blocks as well as gradual after effects in washout blocks. We

478 therefore performed an analysis similar to the savings analysis in Figure 4, and calculated the

479 time constants for the washout and learning blocks for the "full learners" and "partial learners"

480 sub-groups (as defined by the $80 \%$ threshold discussed above) for all the four $20^{\circ}$ perturbation

481 conditions (groups 1a, 1b, 2a, and 2b). This resulted in 33 full learners and 10 partial learners.

482 Figure 7 shows that indeed, the full-learners showed large savings and short after-effects,

483 whereas the partial-learners showed little savings and strong after-effects. The overall time

484 constants of relearning blocks (mean of LB2, LB3, and LB4) of the full-learners were

485 significantly smaller than those of the partial-learners (Wilcoxon-Mann-Whitney Test; $W=$ 
530, $p=0.0001$ ), despite the fact that their initial learning was not significantly different

487 (comparing LB1; $W=358, p=0.62$ ). In addition, the overall time constants of washout

488 blocks (mean of WB1, WB2, and WB3) of the full-learners were significantly smaller than those 489 of the partial-learners $(W=542, p=0.00005)$.

491 Savings and aftereffects following gradual and abrupt perturbations: Simulation 492 results

493 Here we present simulations that account for "one-trial" savings in the data from Roemmich and 494 Bastian (2015). Specifically, these authors showed that whereas a gradual perturbation followed 495 by a washout period does not lead to savings in a subsequent abrupt re-adaptation phase (their 496 phase G1WA2), an abrupt perturbation followed by a washout period leads to large savings in a 497 subsequent abrupt re-adaptation phase (AWA2). A short abrupt perturbation followed by a 498 washout period also leads to savings in a subsequent abrupt re-adaptation phase (sAWA2).

499 Figure 8A (left) shows the hand direction in the three conditions for a single simulation runs.

500 Figure $8 \mathrm{~B}$ shows the average of 50 simulations, in which, similar to Figure 4 in Roemmich and 501 Bastian (2015), we superimposed the adaptation A2 in G1WA2 to the initial abrupt adaptation A 502 in AWA2 (top), the adaptation A2 in AWA2 to the initial abrupt adaptation A in AWA2

503 (middle), and the adaptation A2 in sAWA2 to the initial abrupt adaptation A in AWA2 bottom.

504 As in previous experiments in locomotion (Roemmich and Bastian, 2015) and arm movements

505 (Herzfeld et al., 2014), the gradual adaptation GWA2 resulted in no savings. In contrast, the 506 initial abrupt adaptation in AWA2 resulted in large "one-trial" savings and, to a lesser extent, the 507 short initial abrupt adaptation in AWA2 also resulted in savings. 


\section{Discussion}

509 The significance of our study is that we propose a unifying model of motor adaptation that

510 accounts for a variety of phenomena that appear, a priori, contradictory. Our key novel insight is

511 that qualitative differences in learning, washout, savings, and decay in error-clamp between

512 conditions and between participants are all linked and explained by a single computational model

513 that uses a stochastic Bayesian decision-making process. At every trial, the sensory prediction

514 error of the internal models will determine whether i) a previously updated expert model is

515 recalled and updated, ii) a novice model is selected and develop into a new expert, or iii) the

516 baseline model is updated. Because of these three different modes of operations, the model

517 generates qualitative differences in savings and during error clamps.

\section{Savings following large vs. small perturbations and abrupt vs. gradual}

\section{0 perturbations}

521 As predicted by the model, experimental data revealed that savings were dominant in the large,

522 but not in the small, perturbation conditions. In contrast, long-lasting after-effects were observed

523 in the small, but not in the large, perturbation condition. These results partially replicate those of

524 Morehead et al. (2015), with the main difference that we used $20^{\circ}$ and $10^{\circ}$ for the large and small

525 perturbation with a single target, whereas they used $45^{\circ}$ and $15^{\circ}$ with two targets. In addition, our

526 results are in line with those of previous studies showing that savings in motor adaption depend

527 on the condition of the perturbation. On one hand, savings occur after an initial large abrupt

528 perturbation followed by a washout period (Klassen et al., 2005; Krakauer et al., 2005; Morehead

529 et al., 2015), even with very few trials of adaptation (Huberdeau et al., 2015), and after a gradual 25 
530 perturbation if followed by a no-activity period (Klassen et al., 2005). On the other hand, no

531 savings occur when a gradual adaptation is followed by a washout period (Herzfeld et al., 2014;

532 Roemmich and Bastian, 2015), unless there was a large error at the end of the gradual adaptation

533 block (Turnham et al., 2012).

534 Previous models cannot account for these various savings results. Linear time-invariant models

535 can only account for savings following very short washout sessions (Smith et al., 2006). Models

536 with multiple memories can account for savings following abrupt perturbations because they

537 protect existing memories. However, the Lee and Schweighofer (2009) model needed an external

538 contextual cue for long-term update, and thus cannot account for savings after gradual

539 adaptation, and the Berniker and Kording (2011) model predicted savings following gradual

540 adaptation, unlike the data of Roemmich and Bastian (2015). In contrast, our current model

541 explains these results because only the baseline model is updated during gradual adaptation.

\section{Stochastic lags versus gradual decay in error-clamp}

544 Our model and experimental results allow reconciliation of previously controversial

545 experimental results on error-clamps. It has been shown that performance during clamp can be

546 sustained near the perturbation level and decay stochastically after varying lag durations (Scheidt

547 et al., 2000; Vaswani and Shadmehr, 2013; Vaswani et al., 2015). In stark contrast, Brennan and

548 Smith (2015) proposed that the lag observed in the Vaswani and Shadmehr (2013) study was an

549 artifact, and that the decay in error-clamp was due to trial-by-trial passive forgetting. Our data

550 show both lags and gradual decay, with the conditions of adaptation controlling the decay

551 behavior in the clamp. Large perturbation amplitudes and low noise levels promote long 
552 stochastic lags followed by abrupt decay. Large amplitude and large noise levels promote shorter

553 lags. Small amplitude or gradual adaptation promotes gradual decay. In addition, following large

554 perturbations, subjects showing higher adaptation levels exhibit lags, whereas subjects showing

555 lower levels exhibit gradual decay.

556 The distinction between two-model learners and one-model learners can reconcile these error-

557 clamp data. If the learner becomes a two-model learner, then lags and model switching based on

558 prediction error occurs. Contrarily, if the learner only updates the baseline model, then passive

559 gradual decay occurs. In addition, increase in motor or sensory noise leads to greater noise in

560 sensory prediction error, and therefore increasing chance of switching to the baseline model,

561 with shorter lags. To demonstrate that switching can occur in error-clamp, we inserted trigger

562 trials in the error-clamp following adaptation to large perturbation. The data often showed fast

563 switches and sustained performance just after these single trials. Some subjects did not respond

564 to trigger trials. However, these subjects were partial learners, which suggests that they only

565 updated their baseline models.

566 Recent research (Taylor et al., 2014) revealed that adaptation has both a "strategic" and implicit

567 components. Strategic learning is temporally-stable, while implicit learning consists of both

568 temporally-stable and temporally-labile components (Miyamoto et al., 2014). Involvement of

569 such strategic learning could explain why, in our simulations, a larger decay rate was needed in

570 the baseline model than in the expert models to account for decay in the clamp data (large decay

571 rate in the expert models would have created much shorter lags). This suggests that update of a

572 newly learned model involves both strategic (temporally-stable) and implicit (both stable and

573 labile) learning, whereas update of the baseline model mostly involves implicit learning. 


\section{$575 \quad$ Limitations and future work}

576 A first limitation of our model is that it accounts for only one of the two types of savings

577 (Roemmich and Bastian, 2015), the initial savings shown in the first trials of re-adaptation. Later 578 savings, seen by an increase in learning rate require a meta-learning process, e.g., (Schweighofer 579 and Doya, 2003; Herzfeld et al., 2014). Future models will need to incorporate these two types of 580 savings to provide a full account of existing experimental data. A second limitation is that we 581 assumed, for the sake of simplicity, that only prediction errors update motor adaptation.

582 However, motor adaptation is also updated by reward-based and use-dependent mechanisms, 583 e.g., (Huang et al., 2011; Shmuelof et al., 2012; Galea et al., 2015). Because such additional 584 mechanism influence savings (Huang et al., 2011), dissociation of the multiple different factors

585 that influence savings is needed. A third limitation is that we did not model processes with 586 different timescales involved in motor adaptation. Previous studies have shown the existence of 587 fast and slow processes (related to the temporally stable and labile processes discussed 588 above)(Smith et al., 2006), possibly updated in parallel (Lee and Schweighofer, 2009); recent 589 studies suggest that additional time processes exist (Kording et al., 2007; Kim et al., 2015).

590 Future work will be needed to understand how to integrate these fast/slow processes with the 591 multiple memories framework discussed in this paper.

\section{Related models}

594 Our main question was to study how the CNS creates new internal models (Shadmehr and

595 Mussa-Ivaldi, 2012). The question of updating existing models been addressed via the mixture of 596 experts' models, e.g., (Jordan and Jacobs, 1991; Ghahramani and Wolpert, 1997; Wolpert and 
Kawato, 1998; Haruno et al., 2001; Lee and Schweighofer, 2009; Schweighofer et al., 2011; Lee et al., 2016). While these previous models used experts to explain adaptation to multiple tasks, the current model and a previous model (Berniker and Kording, 2011) suggest that modular experts are needed to explain both learning and forgetting of a single task. experts a priori, we assumed here the existence of novice models. Similarly, Lonini et al. (2009) proposed that new local regression models are added whenever a new data point does not

604 activate any of the existing models above a threshold. As in our model, previously learned 605 memories are protected, thus the model can show savings. However, it is unclear, how their model could account for data with small or gradual adaptation and for the qualitative difference

607 in error-clamps.

608 Our model is highly related to a previous model of visual memory (Gershman et al., 2014). Like 609 our model of motor adaptation, this model is based on a bank of Kalman filters, and new 610 memories are created when discontinuities in sensory data cannot be explained by existing 611 memories. Thus, it is striking that a similar mechanism, based on prediction errors, may be

612 involved in the formation and update of different types of memories (visual memory and motor 613 memory) in presumably different brain areas, with the cerebellum involved in visuomotor

614 memories, e.g., (Kim et al., 2015). Thus, our simulation and behavioral data of motor adaptation

615 are in line with the general view of human learning according to which new memories are

616 created when no existing memories can account for discontinuities in sensory data (Gershman et 617 al., 2014).

618 In these models, the width of the "receptive field" of the prediction error determines if the new 619 data is sufficiently "close" to the memory. We proposed that the width of the baseline model is 
620 subject-specific. Accordingly, what constitute a large or a small perturbation is variable between

621 subjects and may depend on the uncertainty of the baseline model being within "natural error

622 range" (Torres-Oviedo and Bastian, 2012). Additional studies are needed to determine the neural

623 correlates underlying one-model versus two-model learning in motor adaptation.

624

625

626

627

628

629

630 


\section{References}

632

633

634

635

636

637

638

639

640

641

642

643

644

645

646

647

648

649

650

651

652

653

654

655

656

657

658

659

660

661

662

663

664

665

666

667

668

669

670

671

672

673

Berniker M, Kording KP (2011) Estimating the relevance of world disturbances to explain savings, interference and long-term motor adaptation effects. PLoS Comput Biol 7:e1002210.

Bertin M, Schweighofer N, Doya K (2007) Multiple model-based reinforcement learning explains dopamine neuronal activity. Neural Netw 20:668-675.

Bishop G, Welch G (2001) An introduction to the Kalman filter. In: SIGGRAPH, Course, pp 27599-23175.

Brennan AE, Smith MA (2015) The Decay of Motor Memories Is Independent of Context Change Detection. PLoS Comput Biol 11:e1004278.

Cothros N, Wong JD, Gribble PL (2006) Are there distinct neural representations of object and limb dynamics? Exp Brain Res 173:689-697.

Doya K, Samejima K, Katagiri K, Kawato M (2002) Multiple model-based reinforcement learning. Neural computation 14:1347-1369.

Galea JM, Mallia E, Rothwell J, Diedrichsen J (2015) The dissociable effects of punishment and reward on motor learning. Nat Neurosci 18:597-602.

Gershman SJ, Radulescu A, Norman KA, Niv Y (2014) Statistical computations underlying the dynamics of memory updating. PLoS Comput Biol 10:e1003939.

Ghahramani Z, Wolpert DM (1997) Modular decomposition in visuomotor learning. Nature 386:392-395.

Haruno M, Wolpert DM, Kawato M (2001) Mosaic model for sensorimotor learning and control. Neural computation 13:2201-2220.

Herzfeld DJ, Vaswani PA, Marko MK, Shadmehr R (2014) A memory of errors in sensorimotor learning. Science 345:1349-1353.

Huang VS, Haith A, Mazzoni P, Krakauer JW (2011) Rethinking motor learning and savings in adaptation paradigms: model-free memory for successful actions combines with internal models. Neuron 70:787-801.

Huberdeau DM, Haith AM, Krakauer JW (2015) Formation of a long-term memory for visuomotor adaptation following only a few trials of practice. J Neurophysiol 114:969977.

Izawa J, Shadmehr R (2011) Learning from sensory and reward prediction errors during motor adaptation. PLoS Comput Biol 7:e1002012.

Jordan MI, Jacobs RA (1991) A competitive modular connectionist architecture In: Advances in neural information processing systems pp 767-773.

Kawato M, Wolpert D (1998) Internal models for motor control. Novartis Found Symp 218:291304; discussion 304-297.

Kim S, Ogawa K, Lv J, Schweighofer N, Imamizu H (2015) Neural Substrates Related to Motor Memory with Multiple Timescales in Sensorimotor Adaptation. PLoS Biol 13:e1002312.

Klassen J, Tong C, Flanagan JR (2005) Learning and recall of incremental kinematic and dynamic sensorimotor transformations. Exp Brain Res 164:250-259.

Kluzik J, Diedrichsen J, Shadmehr R, Bastian AJ (2008) Reach adaptation: what determines whether we learn an internal model of the tool or adapt the model of our arm? $\mathrm{J}$ Neurophysiol 100:1455-1464. 
Kording KP, Tenenbaum JB, Shadmehr R (2007) The dynamics of memory as a consequence of optimal adaptation to a changing body. Nat Neurosci 10:779-786.

Korenberg AT, Ghahramani Z (2002) A Bayesian view of motor adaptation. Current Psychology of Cognition 21:537-564.

Krakauer JW, Ghilardi MF, Ghez C (1999) Independent learning of internal models for kinematic and dynamic control of reaching. Nat Neurosci 2:1026-1031.

Krakauer JW, Ghez C, Ghilardi MF (2005) Adaptation to visuomotor transformations: consolidation, interference, and forgetting. J Neurosci 25:473-478.

Lee JY, Schweighofer N (2009) Dual adaptation supports a parallel architecture of motor memory. J Neurosci 29:10396-10404.

Lee JY, Oh Y, Kim SS, Scheidt RA, Schweighofer N (2016) Optimal Schedules in Multitask Motor Learning. Neural computation 28:667-685.

Lee K, Oh Y, Izawa J, Schweighofer N (2018) What you predict, not what you do, updates memories in visuomotor adaptation. bioRxiv 313551.

Lonini L, Dipietro L, Zollo L, Guglielmelli E, Krebs HI (2009) An internal model for acquisition and retention of motor learning during arm reaching. Neural computation 21:2009-2027.

Mazzoni P, Krakauer JW (2006) An implicit plan overrides an explicit strategy during visuomotor adaptation. J Neurosci 26:3642-3645.

Miyamoto YR, Wang S, Brennan AE, Smith MA (2014) Distinct forms of implicit learning that respond differentially to performance errors and sensory prediction errors. In: Transl. Comput. Mot. Control (TCMC).

Morehead JR, Qasim SE, Crossley MJ, Ivry R (2015) Savings upon Re-Aiming in Visuomotor Adaptation. J Neurosci 35:14386-14396.

Roemmich RT, Bastian AJ (2015) Two ways to save a newly learned motor pattern. J Neurophysiol 113:3519-3530.

Scheidt RA, Reinkensmeyer DJ, Conditt MA, Rymer WZ, Mussa-Ivaldi FA (2000) Persistence of motor adaptation during constrained, multi-joint, arm movements. J Neurophysiol 84:853-862.

Schweighofer N, Doya K (2003) Meta-learning in reinforcement learning. Neural Netw 16:5-9.

Schweighofer N, Lee JY, Goh HT, Choi Y, Kim SS, Stewart JC, Lewthwaite R, Winstein CJ (2011) Mechanisms of the contextual interference effect in individuals poststroke. $\mathbf{J}$ Neurophysiol 106:2632-2641.

Shadmehr R, Mussa-Ivaldi S (2012) Biological learning and control: how the brain builds representations, predicts events, and makes decisions. : Mit Press.

Shmuelof L, Huang VS, Haith AM, Delnicki RJ, Mazzoni P, Krakauer JW (2012) Overcoming motor "forgetting" through reinforcement of learned actions. J Neurosci 32:14617-14621.

Smith MA, Ghazizadeh A, Shadmehr R (2006) Interacting adaptive processes with different timescales underlie short-term motor learning. PLoS Biol 4:e179.

Taylor JA, Ivry RB (2011) Flexible cognitive strategies during motor learning. PLoS Comput Biol 7:e1001096.

Taylor JA, Krakauer JW, Ivry RB (2014) Explicit and implicit contributions to learning in a sensorimotor adaptation task. J Neurosci 34:3023-3032.

Torres-Oviedo G, Bastian AJ (2012) Natural error patterns enable transfer of motor learning to novel contexts. J Neurophysiol 107:346-356.

Turnham EJ, Braun DA, Wolpert DM (2012) Facilitation of learning induced by both random and gradual visuomotor task variation. J Neurophysiol 107:1111-1122. 
Vaswani PA, Shadmehr R (2013) Decay of motor memories in the absence of error. J Neurosci 33:7700-7709. Krakauer JW (2015) Persistent residual errors in motor adaptation tasks: reversion to baseline and exploratory escape. J Neurosci 35:6969-6977. 


\section{$730 \quad$ Figure legends}

731 Figure 1. (A) The mixture of experts' framework. We assumed that the central nervous system

732 maintains a baseline model with relatively low uncertainty and a number of perturbation models.

733 Each model $i$ maintains and updates a perturbation mean $\hat{p}_{t}^{i}$ and uncertainty $\hat{S}_{t}^{i}$. Combination of

734 the model priors and likelihood generates a model weight $w_{t}^{i}$ after normalization by the "gating

735 controller". The weight has two main roles: it controls both the motor output and the model

736 updates. (B) Decision in visuomotor adaptation. Top: because the perturbation amplitude is large

737 with respect to decision boundaries, the learner develops and updates a new expert "Perturbation

738 model". Bottom: because the perturbation is small, it simply updates the existing "Baseline

739 model". The light red and blue curves in each panel represent gradual model updates.

741 Figure 2. Experiment design. (A) Diagram showing the three stages of the visuomotor rotation

742 paradigm: baseline and washout, adaptation, and error-clamp. Black lines indicate hand

743 movements (occluded to the subjects) and red lines indicate cursor movements. Red curves

744 around the cursor lines indicate the added Gaussian noises to the cursor in experiments. Left

745 panel- baseline and washout: no rotation besides Gaussian noise. Middle panel - visuomotor

746 rotation; the hand movement illustrates behavior at the end of adaptation. Right panel - visual

747 error-clamp: the cursor feedback location is independent of hand direction. (B) The five

748 experimental conditions. Subjects in all conditions experienced the same perturbation schedules

749 except for perturbation magnitude and perturbation noise level. Black lines in adaptation and

750 washout blocks indicate actual rotation, after Gaussian noise added. Red lines in error-clamp 
751 indicate actual visual feedback provided. The two black vertical lines in the error clamp show

752 single perturbation ("trigger") trials given in conditions $1 \mathrm{~b}$ and $2 \mathrm{~b}$.

754 Figure 3. Examples of simulation run for each of the five experimental conditions. First column:

755 black line - negative hand direction, gray line - rotation, gray area - error-clamp block, red

756 dashed line - trigger trial. Second column: perturbation estimation of each learning model.

757 Subscript 0 represents the baseline model (blue), and subscript 1 represents the perturbation

758 model or the novice model if not updated (red). Third column: sum of the two model's

759 likelihoods (divided by two). Notice how, in the first four conditions, the likelihood of the

760 perturbation model increases as this model better predicts the perturbation, via both the mean

761 prediction convergence to the perturbation level and the decrease in model uncertainty. Fourth

762 column: weight map of the perturbation model, drawn as a function of hand angle and trial. Light

763 gray lines indicate the corresponding weight.

765 Figure 4. Savings and washout in large and small perturbation conditions. (A-C) Upper row:

766 Conditions $1 \mathrm{a}$ and $1 \mathrm{~b}\left(20^{\circ}\right.$ rotation). Bottom row: Condition 3 (10 rotation). (A) Condition-

767 averaged hand direction. Shaded areas around the mean curves (black lines) represent one standard

768 deviation. LB: Learning block (perturbation on), WB: Washout block (perturbation off). (B) Mean

769 time constants for individual exponential fitting from experimental data. Error bars represent

770 standard error. The $* * *$ indicate $\mathrm{p}<0.001$ (Bonferroni-corrected). (C) Estimated time constants for

771 each block from simulated data. 
773 Figure 5. Hand direction in error-clamp. (A) Condition 1a, large perturbation $\left(20^{\circ}\right)$ and small

774 noise $\left(0.5^{\circ}\right)$ : Shaded area represents \pm 1 standard deviation around the condition-averaged plot.

775 (B) Condition 2a, large perturbation $\left(20^{\circ}\right)$ and large noise $\left(4.0^{\circ}\right)$. (C) Condition 3 , small

776 perturbation $\left(20^{\circ}\right)$ and small noise $\left(0.5^{\circ}\right)$. (D) Density plot of hand direction in error-clamp for

777 each condition. The left panel shows experimental data, and the right panel shows simulated

778 data.

779

Figure 6. Hand direction in error-clamp with triggers. Red vertical dotted lines indicate a single

781 trigger (perturbation) trial embedded in error-clamp. (A) Condition 1b, large perturbation $\left(20^{\circ}\right)$

782 and small noise $\left(0.5^{\circ}\right)$. Shaded area represents \pm 1 standard deviation around the condition-

783 averaged plot. (B) Condition $2 \mathrm{~b}$, large perturbation $\left(20^{\circ}\right)$ and large noise $\left(4.0^{\circ}\right)$. (C) Effect of the

784 triggers for the 21 subjects of groups $1 \mathrm{~b} \& 2 \mathrm{~b}$. The X-axis represents the mean hand direction of

7855 trials before each trigger trial, and the Y-axis represents the change in mean hand direction

786 (i.e., mean of 5 trials after trigger minus mean of 5 trials before trigger). Left panel (Data) - each

787 circle represents a single trigger event; since there are 2 triggers for each subject, there are 42

788 events (circles). The red circles indicate trigger events from subjects who adapted to more than

$78980 \%$ of perturbation in the last 10 trials of the last block. The blue circles indicate the other

790 subjects. Right panel (Simulation) - each small circle represents a simulated trigger event from

79150 independent simulation runs. Red circles represent two-model learners, who developed two

792 internal models, whereas blue circles represent one-model learners, who only updated the

793 baseline internal model. 
795 Figure 7. Savings and washout for Full-learners vs. Partial-learners. (A-B) Upper row: Full-

796 learners (Asymptote of LB4 >=16, $\mathrm{N}=33$ ). Partial-learners (Asymptote of LB4 $<16^{\circ}, \mathrm{N}=10$ )

797 (A) Condition-averaged hand direction. Shaded areas around the mean curves (black lines)

798 represent one standard deviation. LB: Learning block (perturbation on), WB: Washout block

799 (perturbation off). Mean time constants for individual exponential fitting from experimental data

800 (B) and simulation data (C). Error bars represent standard errors.

801

802 Figure 8. Simulated savings following gradual (top), abrupt (middle), and short-abrupt (bottom)

803 initial perturbations. A. Single runs illustrating hand directions (Left) and model updates

804 (middle) in the three conditions. B. Average of 50 simulations, in which the initial and second

805 perturbations are aligned at trial 0 . Note how savings are very strong following a long abrupt

806 perturbation, still important following a short-abrupt perturbation, but there are no savings

807 following the gradual perturbation. Compare with Figure 4 in Roemmich and Bastian (2015). 
bioRxiv preprint doi: https://doi.org/10.1101/439406; this version posted October 9, 2018. The copyright holder for this preprint (which was not certified by peer review) is the author/funder, who has granted bioRxiv a license to display the preprint in perpetuity. It is made available under aCC-BY-NC-ND 4.0 International license.

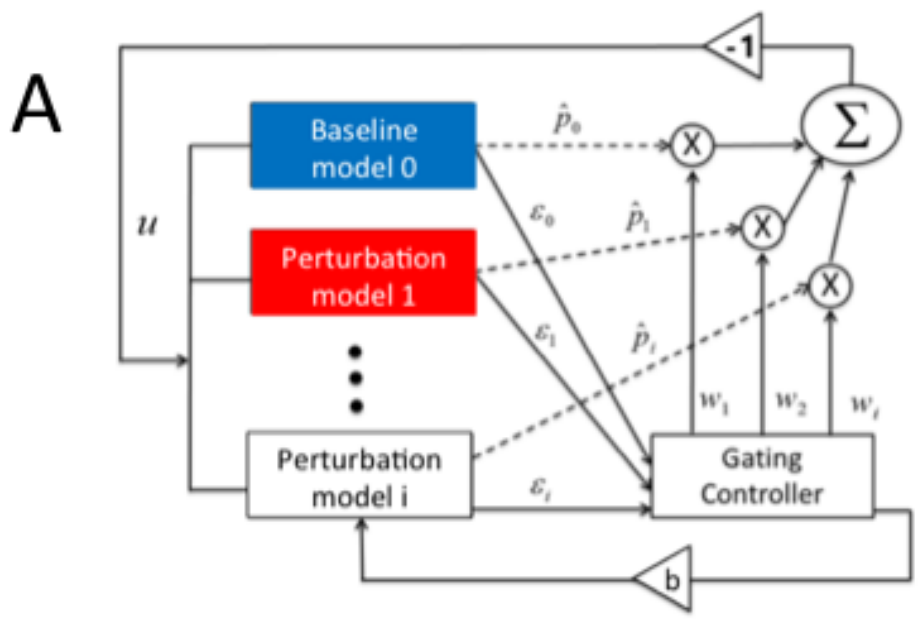

B

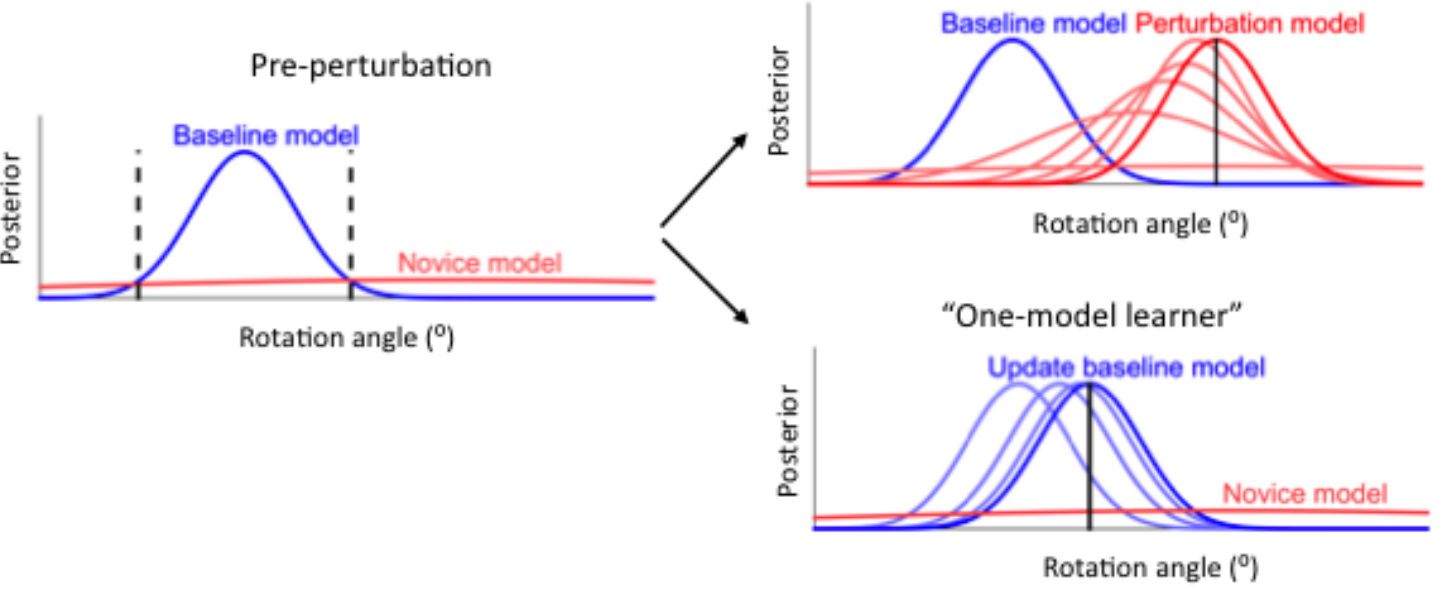

Figure 1. The mixture of experts' framework 
A
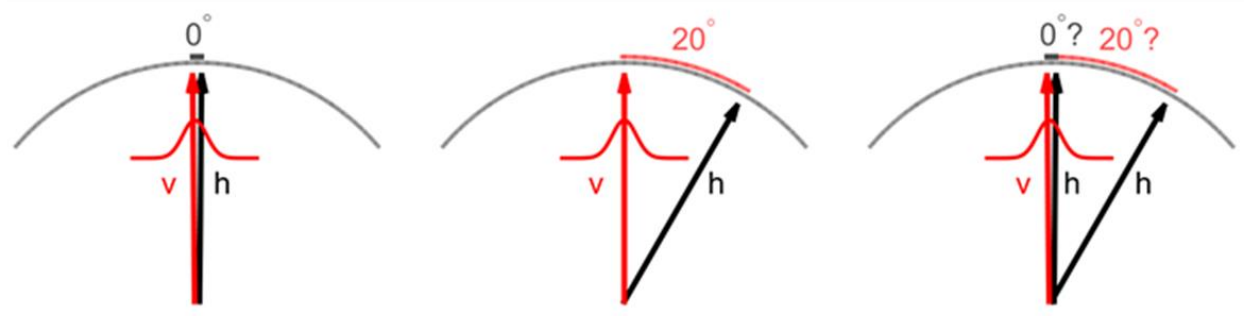

B

Condition $1 \mathrm{a}(\mathrm{n}=11)$ or $1 \mathrm{~b}(\mathrm{n}=11)$

Rotation $20^{\circ} /$ Noise $0.5^{\circ}$

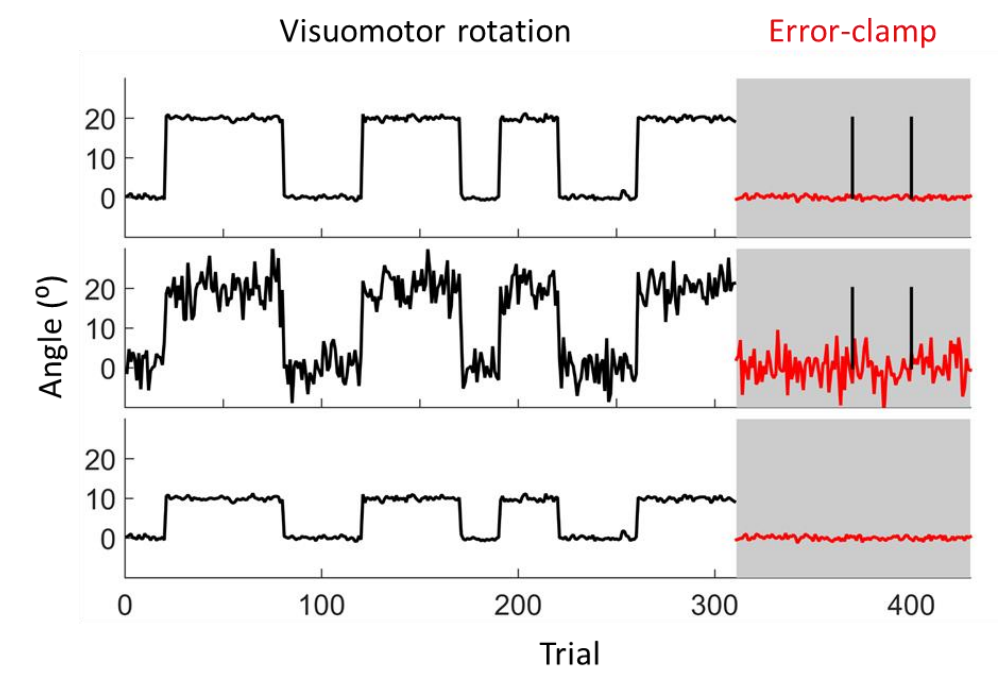

Condition $3(n=11)$ :

Rotation $10^{\circ} /$ Noise $0.5^{\circ}$

Figure 2. Experimental design 
bioRxiv preprint doi: https://doi.org/10.1101/439406: this version posted October 9, 2018. The copyright holder for this preprint (which was not certified by peer review) is the author/funder, who has granted bioRxiv a license to display the preprint in perpetuity. It is made available under aCC-BY-NC-ND 4.0 International license.
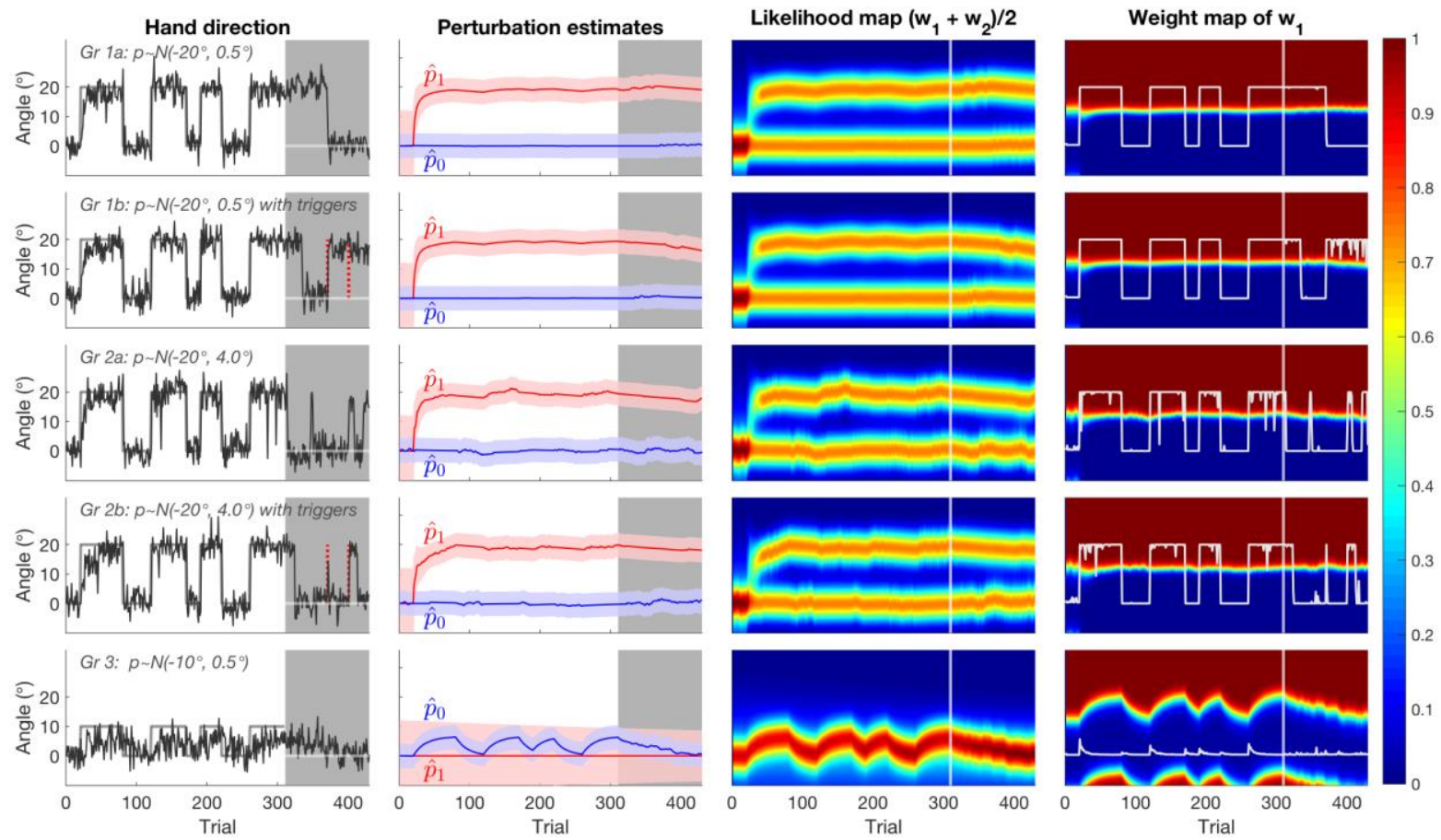

Figure 3. Examples of simulation run for each of the five experimental conditions. 
A

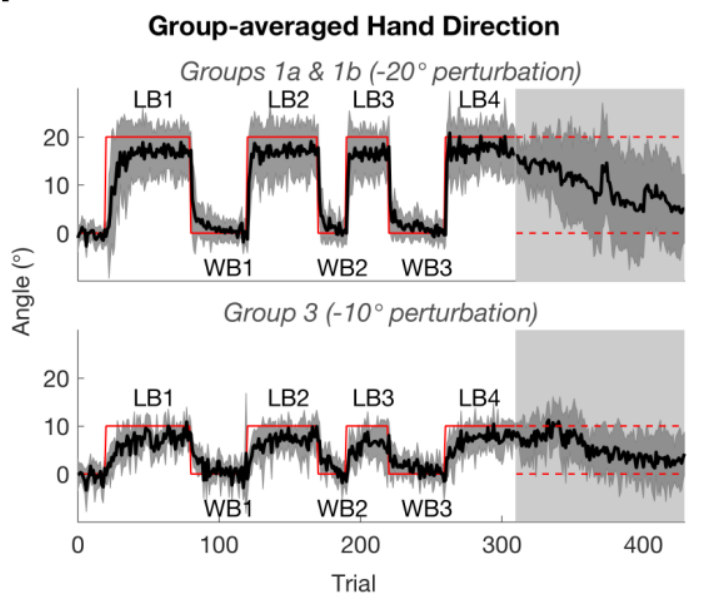

B

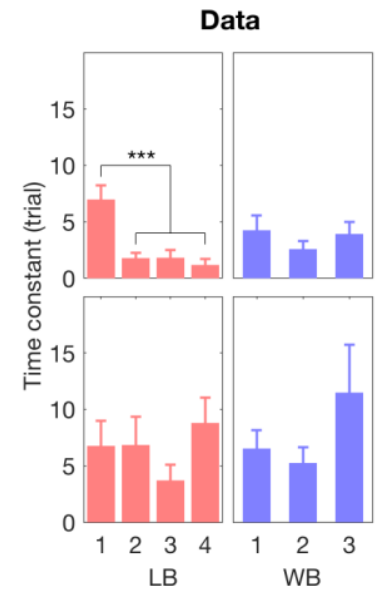

C

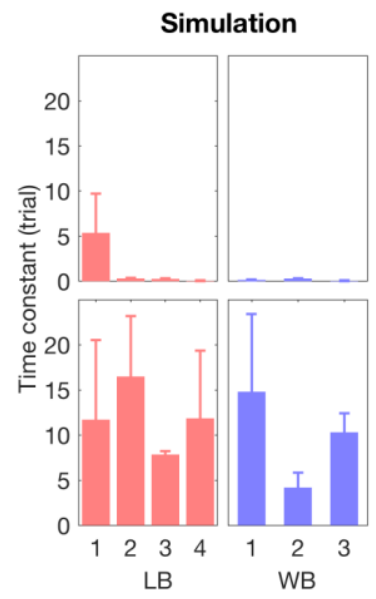

Figure 4. Savings and washout in large and small perturbation conditions. 
bioRxiv preprint doi: https://doi org/10.1101/439406; this version posted October 9 , 2018. The copyright holder for this preprint (which was not certified by peer review) is the author/funder, who has granted bioRxiv a license to display the preprint in perpetuity. It is made available under aCC-BY-NC-ND 4.0 International license.

A
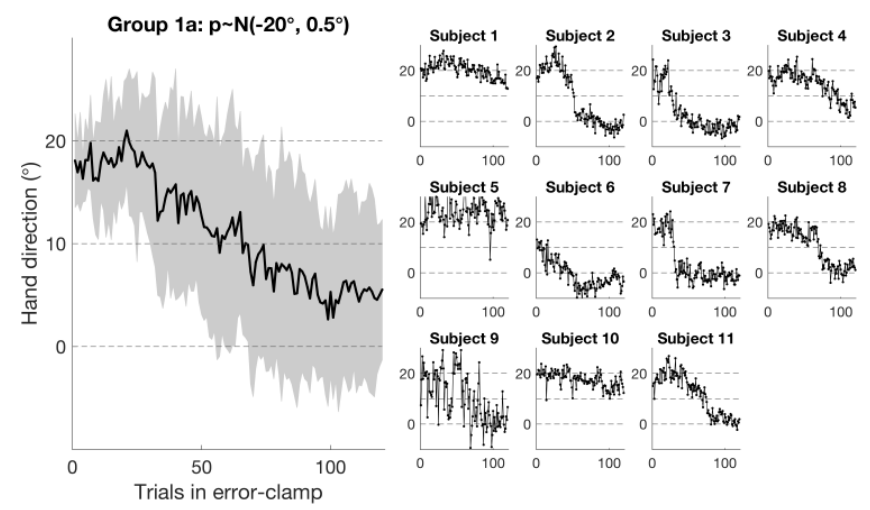

B
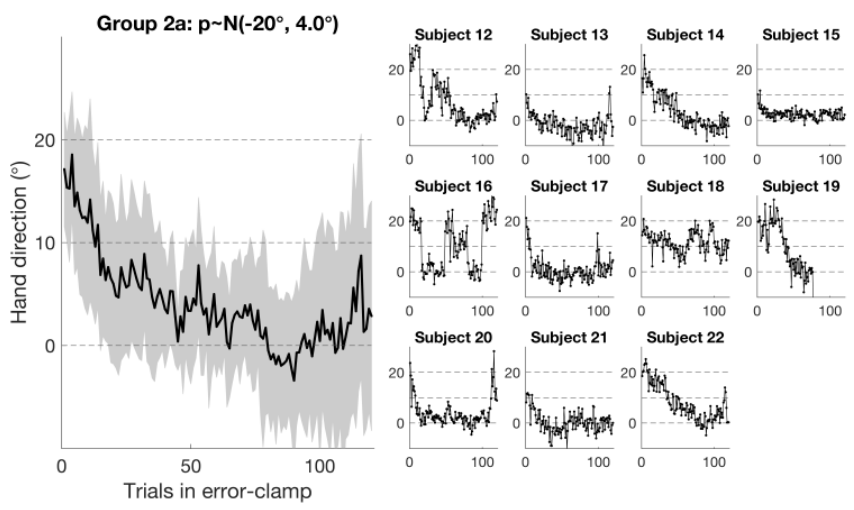

C
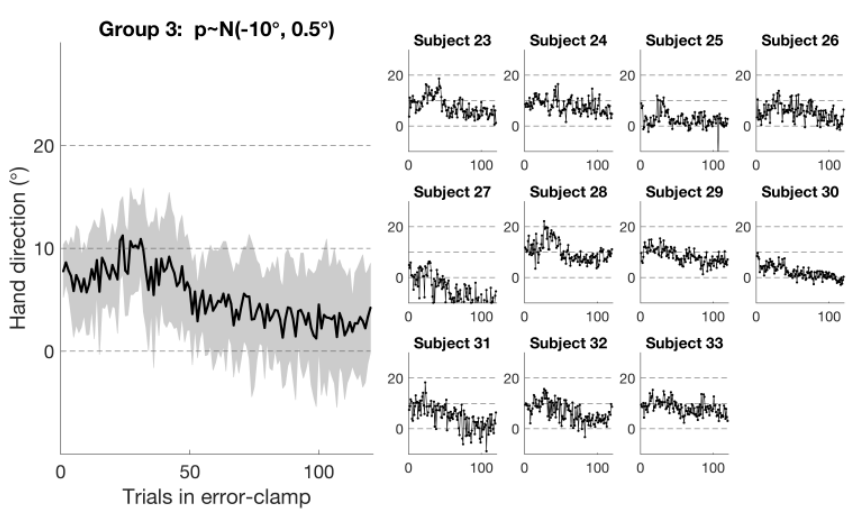

D
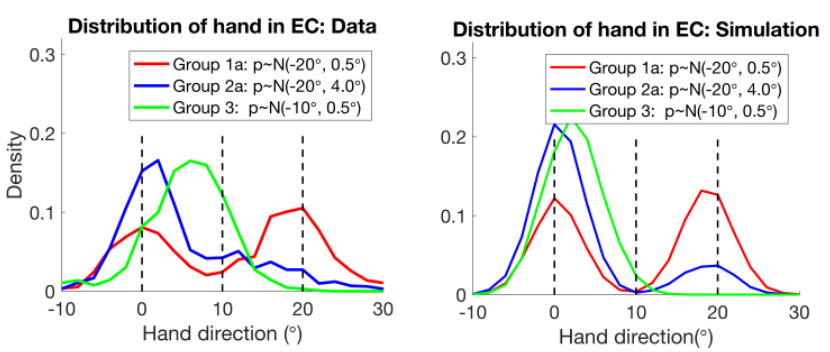

Figure 5. Hand direction in error-clamp. 
bioRxiv preprint doi: https://doi.org/10.1101/439406; this version posted October 9 2018. The copyright holder for this preprint (which was not certified by peer review) is the author/funder, who has granted bioRxiv a license to display the preprint in perpetuity. It is made available under aCC-BY-NC-ND 4.0 International license.

A

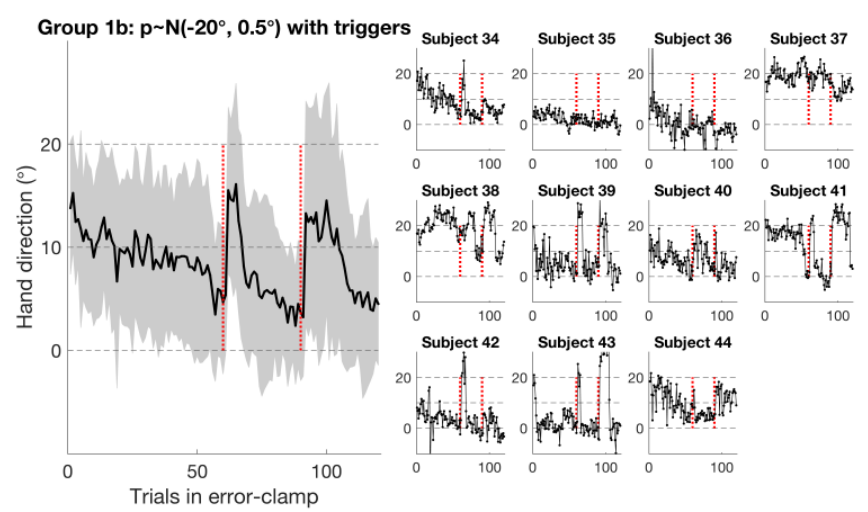

B

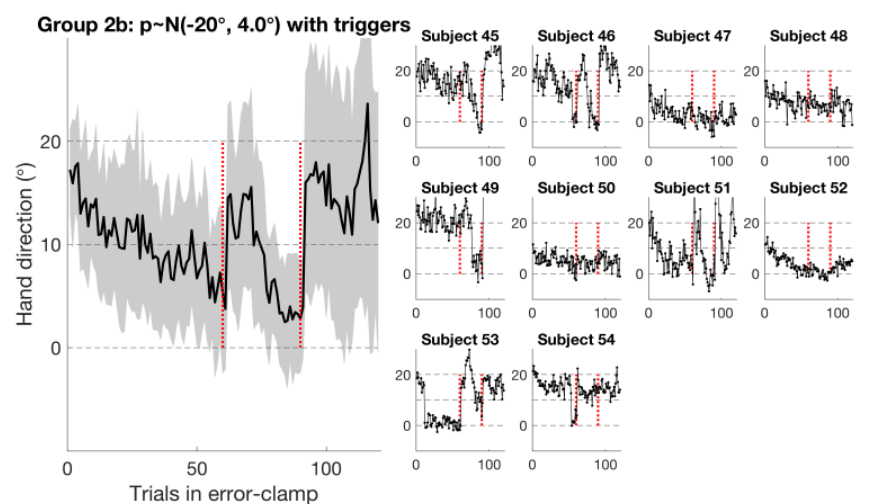

C
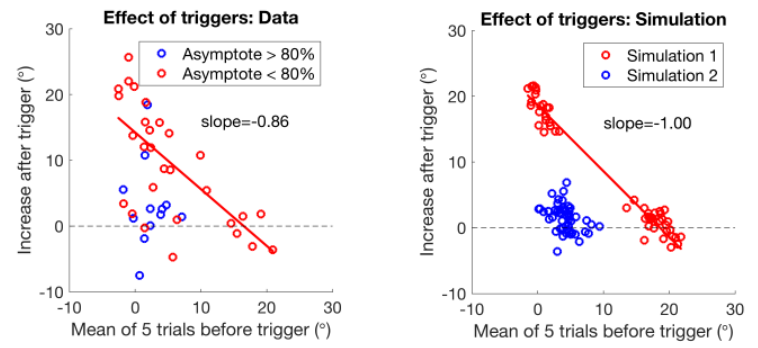

Figure 6. Hand direction in error-clamp with triggers. 
A

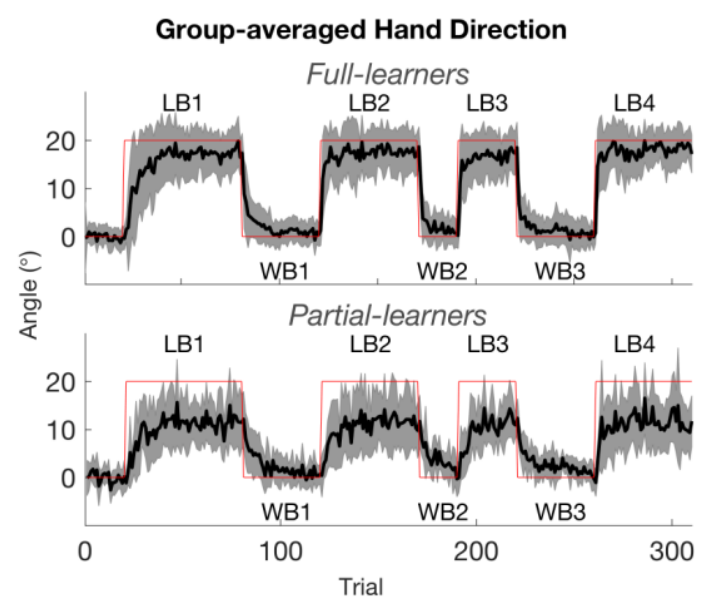

B

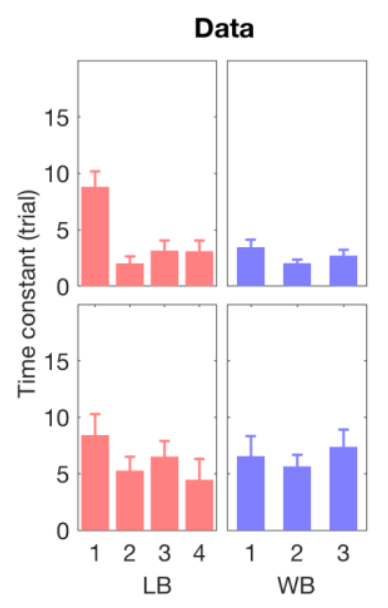

C

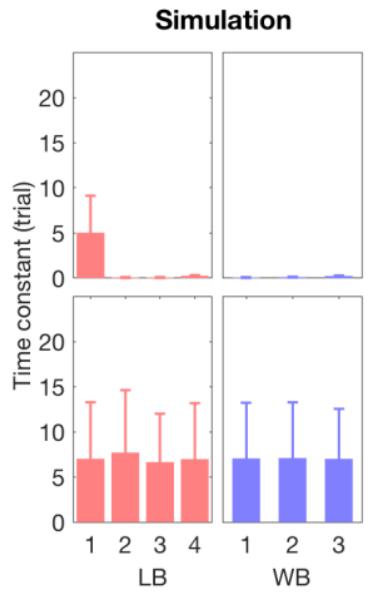

Figure 7. Savings and washout for Full-learners vs. Partial-learners 
A
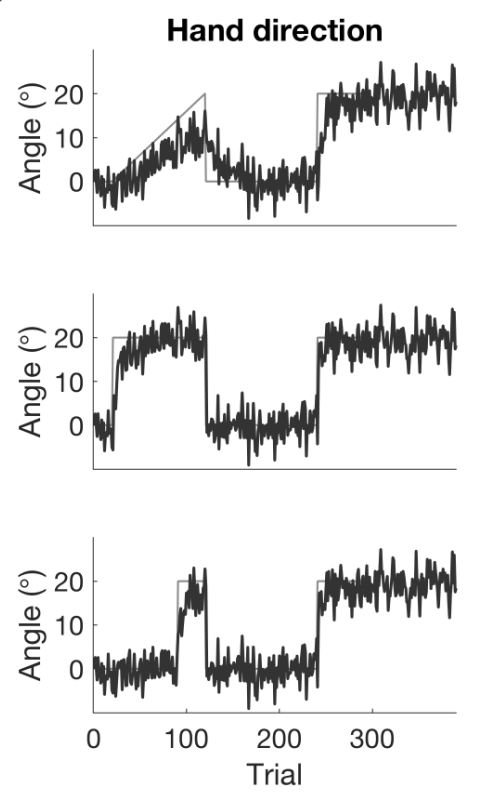

B
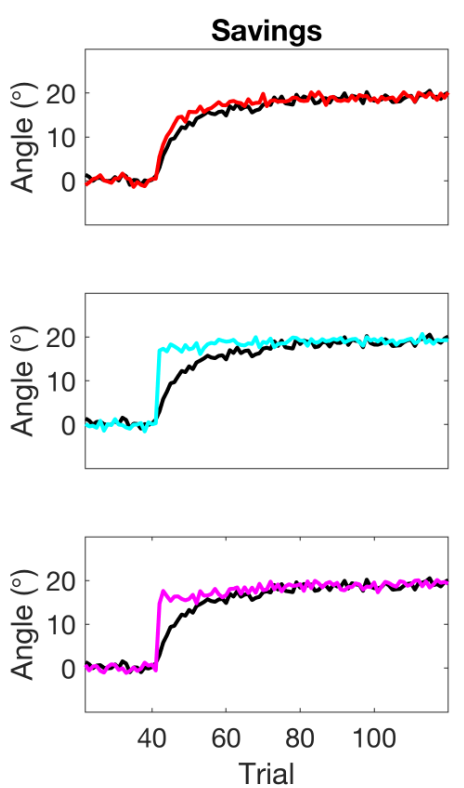

Figure 8. Simulated savings following gradual (top), abrupt (middle), and short-abrupt (bottom) initial perturbations. 